\section{Magnetic resonance spectroscopy}

\author{
Bridging the neurochemistry and neuroanatomy \\ of schizophrenia
}

PETER F. BUCKLEY and LEE FRIEDMAN

The neuroscience of schizophrenia is daunting. While a firm neurobiological substrate has yet to be clearly elucidated, a plethora of pathological brain changes has been observed across molecular, cellular and structural levels. At each juncture, findings are inconsistent across time and between studies. In addition, recent refinements in neuroscience have expanded and intensified the field of enquiry in schizophrenia research to an unprecedented level. Conceptualisations of single neurotransmitter dysfunction have been revitalised and replaced by more complex models of integrative failure across multiple neurotransmitter and neuromodulatory systems. Similarly, 'single-lesion' perspectives of pathomorphology have broadened to encompass notions of diffuse cortical abnormalities that underlie perhaps additional and selective (fronto-temporal) tissue damage in schizophrenia (McCarley et al, 1999). The notion of schizophrenia as a disorder(s) of neural disconnectivity - whether espoused from the vantage point of neurochemistry or of morphometry - is gaining momentum and is intuitively consistent with the complexity of presentations and outcomes for schizophrenia. The challenge to contemporary neuroscience is to harness an everincreasingly sophisticated array of technologies to detail each specific deficit and, thereupon, to integrate these within a cogent schema of the neurobiology of schizophrenia. Simply put, seeing the woods from the trees is not an easy task today in schizophrenia research.

Magnetic resonance spectroscopy (MRS) is a non-invasive technique that provides useful information on brain chemistry. In vivo information on neuronal function is accessible via ${ }^{31} \mathrm{P}$ (phosphorus) and ${ }^{1} \mathrm{H}$ (proton) MRS (Pettegrew et al, 1991; Bertolino et al, 1996). ${ }^{31} \mathrm{P}-\mathrm{MRS}$ allows estimation of phosphomonoesters and phosphodiesters, the building blocks and by-products of lipid metabolism and catabolism, respectively; the extent of cellular energy metabolism (according to adenosine diphosphate [beta-ADP]-adenosine triphosphate [beta-ATP], and inorganic phosphate activity), intracellular $\mathrm{pH}$ (derived from inorganic phosphate estimates) and phosphocreatine activity (reflective of energy and protein catabolism) may also be determined. ${ }^{1} \mathrm{H}-\mathrm{MRS}$ allows estimation of $\mathrm{N}$ acetylaspartate (NAA; a putative neuronal marker), choline (a putative neuronal and extraneuronal marker), creatine (a byproduct of high-energy metabolism and protein catabolism), inositol (an intermediary in phospholipid metabolism), $\gamma$-aminobutyric acid (GABA), and glutamate/ glutamine. Although MRS is not without substantial methodological challenges (see below), this modality is well perched between neurochemistry and structural brain research to provide useful, integrative insights into the neurobiology of schizophrenia. It should be acknowledged at the onset that it is one of several imaging approaches and that the other imaging modalities of functional magnetic resonance imaging (fMRI), positron emission tomography (PET) and single photon emission computed tomography (SPECT), have been critical technologies in the study of brain function in schizophrenia. Moreover, MRS is not a substitute for post-mortem and autoradiographic techniques, which have been extensively applied to schizophrenia research.

\section{MRS AND NEUROCHEMISTRY}

Earlier work demonstrating phospholipid changes in blood and in cerebrospinal fluid (CSF) was curtailed by the inability to examine central nervous system metabolism directly. Current interest in phospholipid metabolism in schizophrenia, particularly as it relates to decreased synthesis and/or increased degradation of the phospholipid-derived membranes in nerve cells, was intensified following publication of the first in vivo ${ }^{31} \mathrm{P}-\mathrm{MRS}$ study of schizophrenia (Pettegrew et al, 1991). In this study of metabolism in the dorsolateral prefrontal cortex of 11 neuroleptic-naïve patients, Pettegrew and colleagues (1991) reported a reduction in phosphomonoesters, a concomitant rise in phosphodiesters, an increase in beta-ATP, and a decrease in inorganic phosphate. These results were interpreted as evidence of membrane instability, with the notion that they might reflect an increase in synaptic pruning at adolescence; this is in accordance with prevailing notions of neurodevelopmental arrest in schizophrenia. Moreover, the proposition that this low phosphomonoester/high phosphodiester perturbation was the biochemical signature of 'neurodevelopmental schizophrenia' was further advanced by a subsequent report (Keshavan \& Pettigrew, 1991) detailing the same phosphomonoester/phosphodiester alteration premorbidly in a 'normal' subject who subsequently developed schizophrenia (and whose MRS profile was identical 2 years later). Stanley et al (1995) reported that an increase in phosphodiesters was evident only in neuroleptic-naïve patients and not in neuroleptic-treated patients with first-episode or chronic schizophrenia; phosphomonoesters were also reduced by $20 \%$ in all groups. In contrast, Volz et al (1998) have noted a decrease in phosphodiesters but no phosphomonoester changes in the frontal cortex of chronic, neuroleptic-treated patients.

Accruing data from post-mortem and immunohistochemical studies now implicate glutamatergic dysfunction in schizophrenia (Tamminga, 1999). Moreover, the rich interconnectivity of glutamatergic efferents from the thalamic and subcortical regions up to the frontal cortex suggest that such dysfunction is unlikely to be limited to a single site. The interest in glutamatergic neurotransmission stems not only from the observations of deficits in this system but also from the importance of glutamate-dopamine interactions and the proposed role of glutamate as a modulator of dopaminergic activity. Alterations in post-synaptic glutamate receptor binding and in messenger ribonucleic acid (mRNA), which collectively suggest deficient glutamatergic neurotransmission, have also been observed (Tamminga, 1999). Glutamate and glutamine resonance appear in the ${ }^{1} \mathrm{H}$-MRS spectrum. Until very recently, however, such information was inaccessible 
since it was not possible to degrade the 'twin' peak of this resonance into its separate glutamate and glutamine components. Now, through the use of short echo sequence acquisition, glutamate-glutamine levels can be examined using ${ }^{1} \mathrm{H}-\mathrm{MRS}$ to illuminate data from other sources regarding the glutamatergic biosynthetic pathway in schizophrenia.

Bartha and colleagues have found elevated glutamine, but not glutamate, levels during ${ }^{1} \mathrm{H}$-MRS of the medial prefrontal cortex of ten neuroleptic-naïve patients with schizophrenia (Bartha et al, 1997). These findings are in agreement with a previous report from this group (Stanley et al, 1996), although in this earlier study the increase in glutamine was confined to a subgroup of medicated patients and was not observed in firstepisode patients. The authors postulated that this rise in glutamine was due to a failure of conversion of glutamine to glutamate early during the glutamatergic biosynthesis. Another study (Choe et al, 1994) found a $25 \%$ elevation in the MRS peak ratio of GABA plus glutamate to creatine in the right prefrontal cortex of 23 patients with chronic schizophrenia who were unmedicated at time of scanning. Collectively, these observations are congruent with a defect in glutamatergic functioning in schizophrenia. Greater resolution of the glutamate peak and of those closely related GABA and glutamine peaks should present the opportunity in future studies to characterise the in vivo interactions between these metabolites. It is also noteworthy that the paradigm of probing metabolic interactions by way of in vivo pharmacological challenges has gained prominence and success in functional brain imaging studies, most notably those involving PET. For example, Breier et al (1997) have examined the impact of amphetamine infusions on dopamine neurotransmission during PET scanning of normal volunteers; amphetamine was observed to be a potent stimulator in vivo of dopamine release. This study illustrates well the potential of functional neuroimaging for the better understanding of the dynamic effects of receptor physiology. Given the recent technical improvements in scanning acquisition and in the quantification of MRS metabolites, it is likely that similar paradigms will be applied in future MRS studies to probe the glutamatergic (and perhaps other) neurotransmitter system.

\section{MRS AND NEURONAL INTEGRITY}

Earlier notions of gross structural brain changes in schizophrenia have given way to the recognition that the pathomorphology of schizophrenia is subtle. Although there is support for selective tissue loss in fronto-temporal and heteromodel association areas (McCarley et al, 1999), recent refinements in the segmentation and postprocessing capabilities of structural MRI have generated consistent evidence for a more generalised loss of cortical tissue, with grey matter being more selectively affected (McCarley et al, 1999). It should be appreciated that these changes are modest and are of the order of a $5 \%$ loss in cortical tissue.

These subtle distinctions have also been subject to scrutiny by ${ }^{1} \mathrm{H}$-MRS and this modality has been able to supply some additional information pertinent to the nature and timing of tissue damage. Most studies have focused on NAA concentration and they report a reduction in NAA in the temporal lobe of patients with schizophrenia (Maier et al, 1995; Bertolino et al, 1996, 1998a; Deicken et al, 1998). Complementary studies suggest that these hippocampal NAA decrements are present in childhood-onset (Bertolino et al, 1998b) and first-episode (Renshaw et al, 1995) schizophrenia. However, a more recent MRS study of 11 first-episode, never-medicated patients with schizophrenia did not find a reduction in NAA in the temporal lobe (Bartha et al, 1999). There is also evidence for a reduction in NAA on the frontal cortex (Buckley et al, 1994; Bertolino et al, 1996, 1998a,b). The extent to which this tissue loss reflects neurodevelopmental hypoplasia or neurodegenerative atrophy may be determined in part by the timing of NAA loss and (as has been more recently proposed by Bertolino et al, 1996) by the relative concentrations of NAA and choline within the MRS spectrum. For the former, evidence of reductions in NAA has been noted early in the course of illness (Renshaw et al, 1995; Bertolino et al, 1998b) and this decrement is comparable in pattern and magnitude to those observed in studies of groups of chronically ill patients. These data provide at least indirect evidence for static (neurodevelopmental) brain changes. Studies of high-risk individuals whose developmental trajectory antedates the onset of psychosis would also illuminate this issue; such work is already in hand.
Since elevated choline has been proposed as a marker for neuronal astrocytosis (i.e. reactive gliosis associated with neurodegenerative, traumatic, or other brain injury), it has been suggested that a decrement in NAA occurring in the absence of an increase in the choline signal is evidence for neurodevelopmental rather than neurodegenerative pathomorphology (Bertolino et al, 1996). Buckley et al (1994) reported low NAA and high choline in male patients with schizophrenia. Maier et al (1995) noted reductions in NAA and choline within the hippocampus of patients with schizophrenia. Renshaw et al (1995) reported low $\mathrm{NAA} /$ creatine and high choline/creatine ratios in first-episode patients. In contrast, three studies (Bertolino et al, 1996, 1998b; Deicken et $a l, 1998)$ report a pattern of low NAA and normal choline in schizophrenia. However, it should also be appreciated that diet can impact choline levels and this should be taken into account in future studies (Bertolino et al, 1998b).

Multi-slice ${ }^{1} \mathrm{H}$-MRS imaging, which allows for simultaneous functional examination across several brain regions (heretofore unattainable using single-voxel MRS which typically focuses on one or two regions), has recently come on-line to schizophrenia research and this approach is helpful in addressing the controversy of global $v$. focal pathology in schizophrenia. In three studies which assessed ${ }^{1} \mathrm{H}-\mathrm{MRS}$ metabolites across multiple cortical and subcortical regions, Bertolino and colleagues (1996, 1998a,b) noted selective frontotemporal deficits. Reduction in NAA was the most consistent finding. Interestingly, in each study this decrement proved to be unrelated to hippocampal volume. Lim et al (1998) reported normal NAA values in the grey matter of patients with schizophrenia, despite an $18 \%$ volume reduction which was selective to the grey matter. In contrast, NAA values were reduced (by $7 \%$ ) in the cortical white matter. The volume of the white matter was similar in patients and in control subjects. The capacity to juxtapose MRS metabolite values with grey/white matter volumetric data is a real strength of this study, and it allows for discrimination of grey-white matter pathomorphology in schizophrenia in greater detail than heretofore. This study suggests that different processes may be acting within discrete tissues. However, another recent single-voxel study found no decrements in NAA or grey-white matter volumes in a small sample of patients (Bartha et al, 
1999). Although yielding discrepant findings, both studies are noteworthy since they signal the evolution of more complex MRS examinations which will complement detailed structural imaging assessments. This combined approach should prove most helpful in distinguishing the extent and pattern of global brain changes in schizophrenia.

\section{METHODOLOGICAL CONSIDERATIONS}

It is important to point out that MRS is not without substantial methodological challenges. Although the MRS spectrum can provide information on several important metabolites, distinguishing between individual resonances is difficult and some resonances (e.g. glutamate-glutamine) have MRS peaks which are broad and overlapping. These challenges can in part be overcome by using MRI machines of high field strength (i.e. 4 Tesla), by lengthening the scanning acquisition time, and by altering other study parameters such as the volume of interest. Absolute quantification (rather than relative ratios) of MRS metabolites is another challenge for spectroscopy research, although refinements are now making this task easier.

An effect of antipsychotic medications upon the MRS spectrum cannot be discounted (Buckley et al, 1994; Heimberg et al, 1998). However, studies which have examined this issue have not found any relationship between neuroleptic dose and MRS metabolites (Buckley et al, 1994). Moreover, other studies have reported differences in MRS metabolites between never-medicated patients and normal subjects (Pettegrew et al, 1991; Bartha et al, 1997). On the other hand, one study (Heimberg et al, 1998) raises the possibility that patients receiving atypical antipsychotics may show a different MRS spectrum from patients who have received older antipsychotics. This will require further research to determine the potential for medication-induced changes in the MRS spectrum. This issue will be of particular relevance as MRS is used to examine longitudinal changes in brain chemistry during the course of the illness. Finally, MRS provides one form of imaging data which is best viewed as complementary to data derived from other sources. MRS takes its place alongside the other imaging modalities of

PETER F. BUCKLEY, MD, LEE FRIEDMAN, PhD, Case Western Reserve University, Cleveland, OH, USA

Correspondence: Peter F. Buckley MD, Associate Professor and Vice Chair for Psychiatry, Case Western Reserve University, III00 Euclid Avenue, Cleveland, OH 44I06, USA. Tel: (330) 467-7I3I ext. II59; Fax: (216) 844-1703

(First received II January 1999, final revision I September 1999, accepted I September 1999)

fMRI, PET and SPECT, and these can provide functional data on blood flow, brain glucose metabolism and neuroreceptor occupancy which are beyond the scope and intent of MRS technique.

Notwithstanding these caveats, MRS is a useful technique of potential to yield in vivo information on key cerebral metabolites. Although much of the focus of current MRS research is on determining the timing and progression (or lack thereof) of metabolite changes during the course of the illness, this modality may also provide information on other important aspects, such as the neurochemistry of violence in psychosis or the neurochemistry of alcohol abuse.

\section{REFERENCES}

Bartha, R., Williamson, P. C., Drost, D. J., et al (1997) Measurement of glutamate and glutamine in the medial prefrontal cortex of never treated schizophrenic patients and healthy controls by proton magnetic resonance spectroscopy. Archives of General Psychiatry, 54, 959-965.

_, Al-Semaan, Y. M., Williamson, P., et al (1999) A short echo proton magnetic resonance spectroscopy study of the left mesial-temporal lobe in first-onset schizophrenic patients. Psychiatry, 45, 1403-14II.

Bertolino, A., Nawroz, S., Mattay, V. S., et al (1996) Regionally specific pattern of neurochemical pathology in schizophrenia as assessed by multislice proton magnetic resonance spectroscopic imaging. American Journal of Psychiatry, 153, 1554-1563.

_ , Callicott, J. H., Elman, l., et al (1998a) Regionally specific neuronal pathology in untreated patients with schizophrenia: a proton magnetic resonance spectroscopic imaging study. Biological Psychiatry, 43, 641-648.

_ , Kumra, S., Callicott, J. H., et al (1998b) Commo pattern of cortical pathology in childhood-onset and adultonset schizophrenia as identified by proton magnetic resonance spectroscopic imaging. American Journal of Psychiatry, 155, 1376-1383.

Breier, A., Su, T. P., Saunders, R., et al (1997) Schizophrenia is associated with elevated amphetamineinduced synaptic concentrations: evidence from a positron emission tomography method. Proceedings of the National Academy of Sciences of the United States of America, 94, 2569-2574.

Buckley, P. F., Moore, C., Long, H., et al (1994) 'H magnetic resonance spectroscopy of the left temporal and frontal lobes in schizophrenia: clinical neurodevelopmental and cognitive correlates. Biological Psychiatry, 36, 792-800.

Choe, B. Y., Kim, K. T., Suh, T. S., et al (1994) 'H magnetic resonance spectroscopy characterization of neuronal dysfunction in drug-naive, chronicschizophrenia. Academic Radiology, I, 211-216.

Deicken, R. F., Zhou, L., Schuff, N., et al (1998) Hippocampal neuronal dysfunction in schizophrenia as measured by proton magnetic resonance spectroscopy. Biological Psychiatry, 43, 483-488.

Heimberg, C., Komoroski, R. A., Lawson, W. B., et al (1998) Regional proton magnetic resonance spectroscopy in schizophrenia and exploration of drug effect. Psychiatry Research Neuroimaging, 83, 105-115.

\section{Keshavan, M. S. \& Pettegrew, J.W. (1991)}

Phosphorus-3I magnetic resonance spectroscopy detects altered brain metabolism before onset of schizophrenia (letter). Archives of General Psychiatry, 48, III2-III3.

Lim, K. O., Adalsteinsson, E., Spielman, D., et a (1998) Proton magnetic resonance spectroscopic imaging of cortical gray and white matter in schizophrenia. Archives of General Psychiatry, 55 346-352.

Maier, M., Ron, M. A., Barker, G. J., et al (1995) Proton magnetic resonance spectroscopy: an in-vivo method of estimating hippocampal neuronal depletion in schizophrenia. Psychological Medicine, 25, I201-1209.

McCarley, R.W., Wible, C. G., Frumin, M., et al (1999) MRI anatomy of schizophrenia. Biological Psychiatry, 45 1099-1119.

Pettegrew, J. W., Keshavan, M. S., Panchalingam, K., et al (1991) Alterations in brain high-energy phosphate and membrane phospholipid metabolism in firstepisode, drug-naive schizophrenics. Archives of General Psychiatry, 48, 563-568.

Renshaw, P. F., Yurgelun-Todd, D. A., Tohen, M., et a (1995) Temporal lobe proton magnetic resonance spectroscopy of patients with first-episode psychosis. American Journal of Psychiatry, 152, 444-446.

Stanley, J. A., Williamson, P. C., Drost, D. J., et a (1995) An in-vivo study of the prefrontal cortex of schizophrenic patients at different stages of illness via phosphorus magnetic resonance spectroscopy. Archives of General Psychiatry, 52, 399-406.

$\ldots, \ldots, \ldots$, et al (1996) An in-vivo proton magnetic resonance spectroscopy study of schizophrenia patients. Schizophrenia Bulletin, 22, 597-609.

Tamminga, C. (1999) Glutamatergic aspects of schizophrenia. British Journal of Psychiatry, 174 (suppl. 37), $12-15$.

Volz, H. P., Rzanny, R., Rossger, G., et al (1998) 31Phosphorus magnetic resonance spectroscopy of the dorsolateral prefrontal region in schizophrenics - a study including 60 patients and 36 controls. Biological Psychiatry, 44, 399-404 